\title{
Water Quality Trading in the Presence of Abatement Cost Sharing
}

\author{
November 16, 2011
}

\begin{abstract}
This paper examines how water quality trading interacts with nonpoint-source abatement cost sharing (e.g., as currently practiced by the National Resource Conservation Service through its Environmental Quality Incentives Program (EQIP)) to promote the participation of nonpoint sources in a water quality market; participation that has thus far been noticeably lacking nationwide. As such, an idealized version of water quality trading is envisioned, where water quality trading and nonpoint cost sharing are treated as complementary policy instruments rather than substitutes. Toward this end, the subgame-perfect equilibrium concept is used to model a "multilateral contracting" relationship between the regulatory authority and nonpoint sources when the regulator has incomplete information about the nonpoint sources' production costs. We characterize ex ante (or second-best) nonpoint abatement levels when the regulator chooses cost-share rates in concert with a water quality market. Numerical analysis indicates that current EQIP cost-share rates would likely be lower and more flexibly determined in the presence of water quality trading.
\end{abstract}




\section{Introduction}

The efficacy of incentives contracts for resolving asymmetric information problems is by now well understood. Generally speaking, the principal (or regulator) pays the agent(s) an informational rent in order to procure a desired (typically ex ante) outcome (Laffont and Tirole, 1993). In the case of multilateral contracting, e.g., a single regulator contracting with two or more agents, the procurement problem extends beyond controlling a single agent's decision problem to designing a game that accounts for the agents' interactions with each other (Bolton and Dewatripont, 2005). ${ }^{1}$ The problem also extends to symmetric (or common) uncertainty regarding nature and the absence of a unique dominant strategy equilibrium. This paper considers a multilateral contracting problem where (1) the agents have hidden information about their respective production costs, (2) the regulator is endowed with the authority to subsidize the agents' costs, and (3) the agents trade their product in a quantity-constrained market. ${ }^{2}$

A good example of this type of game is the idealized control of nonpoint-source (NPS) water pollution in the US through water quality trading (WQT) in the presence of existing NPS abatement cost subsidies. We say "idealized" because to date few successful WQT markets have been established in the US for watersheds that include predominantly NPSs (Breetz, et al., 2004; King and Kuch, 2003; Environomics, 1999; Hoag and Hughes-Popp, 1997; King, 2005; Ribaudo, 2001). Therefore, to promote WQT among NPSs, it may be that policy instruments such as abatement cost subsidization need to be used in a complementary fashion with - rather than as substitutes for - WQT. Since the passage of the 1972 and 1977 Federal Water Pollution Control Act Amendments (the Clean Water Act), approximately 34,000 of the US's water bodies have either remained or become non-compliant with the amendments' standards for drinking water, contact recreation, or aquatic life support (EPA, 2005). The main factor contributing to this noncompliance is "loading" of nutrient- and pesticide-based pollutants from agricultural NPSs, e.g., crop and feedlot operations, through natural runoff and leaching processes (Freeman, 2002; EPA, 2011b). Control of NPS loadings, particularly in agricultural-based watersheds, is therefore a crucial determinant of whether

\footnotetext{
${ }^{1}$ For a concise overview of the mechanism design problem see Mas-Colell, et al. (1995, Chapter 23).

${ }^{2}$ As described in the next section, to address this multifacited problem we adopt the subgame-perfect equilibrium concept, which solves a two-stage game between the agents and the regulator. In the first stage of the game the regulator chooses a fixed cost-share (i.e., subsidy) rate and an initial production allocation for each agent. Given the regulator's choices the agents then choose their preferred production allocations, and therefore how much of the product to trade in the second stage of the game.
} 
compliance with the Clean Water Act will ultimately be achieved on a broad scale nationwide.

WQT is premised on the notion that sources within a given watershed (both point and nonpoint) face different abatement cost structures. Assuming these cost differences are divergent enough, and that a given source's abatement level can be estimated with a reasonable amount of certainty, the overall control costs of meeting a given watershed-wide pollution standard can be minimized if sources facing relatively low control costs effectively abate at levels in excess of their respective, required (or regulated) abatement levels and then sell their excess abatement "credits" to relatively high-cost sources in a formal WQT market. To date, however, the involvement of NPSs in WQT has been virtually non-existent in the US, even in spite of the potential for the National Resource Conservation Service's (NRCS's) existing best management practice (BMP) subsidy program, known as the Environmental Quality Incentives Program (EQIP), to stimulate an interest in trading by effectively reducing NPS abatement costs and thus engendering cost divergence between NPSs and point sources in a given watershed. ${ }^{3}$

This paper characterizes one possible solution to the lack of NPS involvement in WQT in the US by examining how a regulator might ideally subsidize BMPs. As demonstrated in Sections 2 and 3, our characterization is ideal in the sense that it (1) assumes WQT and abatement cost subsidization can be used as complementary policy instruments, (2) requires enough information about individual NPS abatement costs within a given watershed to estimate continuous marginal cost functions, and (3) similarly requires enough information about the watershed's topography, soil types, and hydrology to estimate NPS loadings and delivery ratios (or transfer coefficients). ${ }^{4}$ It also assumes the regulator can choose cost-share rates on an individual NPS basis in an effort to minimize the costs associated with meeting an environmental standard. This seemingly innocuous assumption concerning the breadth of the regulator's choice set in determining NPS-specific costshare rates is at odds with historical evidence in the US. Since the program's inception, EQIP cost-share rates as incurred by the NRCS) have traditionally been set by individual states (or

\footnotetext{
${ }^{3}$ EQIP was reauthorized in the Farm Security and Rural Investment Act of 2002 to inter alia provide financial and technical assistance in support of BMP implementation on eligible agricultural land (NRCS, 2006). This program represents one of only two federal programs currently in place nationwide to encourage voluntary control of nonpoint pollution. The other program is the Conservation Reserve Program (CRP) administered through the US Department of Agriculture. Obviously, regulatory reform of EQIP would be required before it could be used in tandem with WQT.

${ }^{4}$ While the appropriateness of the second assumption remains an open question, the suitability of the third assumption is becoming less so over time. We discuss this issue further in Section 2.2.
} 
in some cases, counties within states) at fixed levels across all NPSs, with fixed caps on total funding, total acreage, or total years (NRCS, 2011a). ${ }^{5}$ For example, in Illinois the subsidy rate for BMPs "not related to livestock" is fixed at 60 or 70 percent (75 percent for limited-resource farmers), with a 400-acre limit on certain practices. The rate for confined livestock operations and grazing land applications is fixed at 50,60, or 70 percent (75 percent for limited-resource farmers) (NRCS, 2011b). In contrast, the model presented in this paper provides a clear economic rationale for endowing the regulator with complete flexibility in choosing its cost-share rates for individual NPSs.

Although the economic literature on NPS pollution is quite extensive, it has until recently focused almost exclusively on the use of penalty schemes such as taxation and fines that account for the uncertainty of NPS loadings. ${ }^{6}$ A nascent strand of this literature has also investigated the properties of WQT as a potential NPS control mechanism. ${ }^{7}$ The main focus of this literature has been to identify optimal trading ratios between NPSs and point sources that incorporate loading uncertainty. To our knowledge, the role of subsidization of BMPs in fostering an efficient allocation of NPS abatement effort via WQT has been explored in only one previous paper - Horan et al. (2004). ${ }^{8}$ In light of recent recognition by a host of WQT practitioners concerning the potential synergies between BMP subsidization and WQT (see for instance Kramer (2003), National Association of Conservation Districts (2003), Hall and Raffini (2005), and Breetz and Fisher-Vanden (2007)), this lack of attention in the literature seems particularly glaring.

Horan et al. (2004) call the complementary BMP subsidization/WQT policy described above "double-dipping," and show under what circumstances double-dipping enhances the efficiency of

\footnotetext{
${ }^{5}$ In Section 2 we define cost-share rate as the rate incurred by a given NPS, which is one minus the rate incurred by the regulator, solely for expository convenience.

${ }^{6}$ Good overviews of this literature are provided in Shortle and Horan (2001) and Tomasi, et al. (1994).

${ }^{7}$ As Shortle and Horan (2001) point out, NPS participation in a WQT market can be based on point-source emissions for NPS input trading or point-source emissions for NPS estimated-loadings trading. In this paper, we focus on the latter. More recent papers related to Shortle and Horan (2001) include Shortle and Horan (2005) and Farrow, et al. (2005).

${ }^{8}$ Isik and Sohngen (2003) consider voluntary, performance-based contracts between point and nonpoint sources to reduce joint loadings, where incentive problems are controlled through joint liability and peer pressure/monitoring. Pushkarskaya (2003) investigates an alternative performance-based mechanism that closely resembles the mechanism discussed in this paper. In Pushkarskaya's case, the regulator's payment to an association of NPSs is not in the form of a cost-share subsidy. Rather, it is a lump-sum payment that increases with the group's abatement level. The association - which is constrained by the hidden actions of its members - subsequently decides on an optimal transfer mechanism. Absent from Pushkarskaya's model is a WQT market, which in this paper's model is the vehicle through which NPSs obtain surplus (profit). In Pushkarskaya's case, the association's surplus is created directly via the regulator's payment.
} 
NPS control. They find that a coordinated policy approach, where cost-sharing rates and WQT parameters are set jointly and simultaneously by the regulator (as they are in this paper), outperforms an uncoordinated approach, where the cost-sharing rates are set first and taken as given when setting the trading parameters. While Horan et al. (2004) focus on the individual NPS's tradeoff between accepting a BMP subsidy and the amount of permits it subsequently has for sale (i.e., the case of endogenously determined, partial double-dipping at the NPS level), this paper focuses on the implications of the (information-asymmetric) relationship between the regulator and the NPS when the NPS fully double-dips. Both papers' results point to the advantages of a joint policy when it comes to promoting the participation of NPSs in programs aimed at controlling NPS nutrient loading.

In this paper, we characterize an ex ante (or second-best) solution (i.e., a solution to the incomplete-information problem concerning the NPS abatement efforts) that arises as a result of cost-sharing in concert with WQT. The extent to which the corresponding first-best solution (i.e., the solution to the full-information problem concerning the NPSs' abatement efforts) is constrained by hidden production cost information is demonstrated both analytically and numerically. These solutions focus primarily on the relationship between the regulator and the NPSs, since common practice is for the regulator to subsidize solely NPS abatement, not the abatement efforts of point sources. As such, the solutions we present in this paper are not socially efficient in the broader context of a WQT market. They may instead be considered constrained socially efficient (which we nevertheless refer to as first best), where the constraint reflects the restriction on the regulator's authority over NPSs, in particular its cost-sharing authority. Although our theoretical approach is premised on the notion of an ideal relationship as it might exist between the regulator and NPSs in the US, the main issues addressed jointly in the paper - cost-sharing and pollution trading - are pertinent to WQT markets as they might arise anywhere in the world. Our overriding goal is to characterize NPS cost-sharing and WQT as a joint policy; a policy reflecting some of the realities of current regulatory practice (i.e., the setting of fixed cost-share rates solely for NPSs) as well as the ideal of a possible joint policy.

The next section lays out the basic contracting model describing the interaction between the regulator and the NPSs, which in turn interact with each other and with point sources in a WQT market. Analytical solutions to the model are provided for both the first- and second-best problems. 
Because the analytical solutions are cumbersome, Section 3 solves the model numerically in order to provide estimates of the relative values of key variables. Section 4 summarizes the paper's main findings.

\section{Water Quality Trading and Abatement Cost Sharing}

\subsection{First-Best Benchmark}

For ease of exposition, we assume a watershed is comprised of two NPSs, denoted 1 and 2. There also exists a point source, denoted $P$. NPSs 1 and 2 interact with the regulator and with $P$ in the following two-stage game. In the first stage, the regulator sets the NPSs' respective cost-share rates, $\beta_{1}$ and $\beta_{2}, 0 \leq \beta_{j} \leq 1, j=1,2$, i.e., the proportions of total abatement cost borne by the NPSs themselves. The assumption of fixed cost-share rates set solely for the NPSs and not for the point source is reflective of current regulatory practice in the US, e.g., the NRCS's EQIP for NPS's. The regulator also sets initial abatement allocations $\bar{a}_{1} \geq 0$ and $\bar{a}_{2} \geq 0$ for the NPSs, respectively, and $\bar{a}_{P} \geq 0$ for $P$, which is reflective of the role a regulator would be required to play if a WQT program were to be implemented. ${ }^{9}$ In essence, the regulator offers NPS $j$ specific contract $\left(\beta_{j}, \bar{a}_{j}\right), j=1,2$ and the point source $P$ specific contract $\left(\bar{a}_{P}\right)$.

In the second stage, the NPSs and point source engage in competitive WQT by selecting their respective abatement efforts, $a_{1}, a_{2}$, and $a_{P}$, respectively, and thus their abatement credits available for sale(in need of purchase), $a_{j}-\bar{a}_{j}>(<) 0, j=1,2$, and $a_{P}-\bar{a}_{P}>(<) 0$, whichever the case may be. The assumption of price-taking behavior implies 1 and 2 are either "representative agents" of the two main types of NPSs present in a wider market, or, as the only existing NPSs, they are induced to price competitively by regulatory policy. Further, the price-taking assumption naturally rules out strategic behavior among the NPSs. ${ }^{10}$

As a result of "moving" after the regulator, the NPSs take their respective subsidy rates and initial allocations as given (as does the point source with respect to its initial allocation). However,

\footnotetext{
${ }^{9}$ In reality, the NRCS would not be responsible for setting the initial abatement allocations for WQT. Instead, this responsibility would lie with state-level department(s) of environmental quality (DEQs), or with the U.S. Environmental Protection Agency (EPA) itself. In the context of our model, the regulatory authority might therefore best be interpreted as a joint agency, incorporating both the NRCS and EPA. This joint agency fully characterizes the relationship between NPS polluters and the regulatory authority.

${ }^{10}$ For analysis of imperfectly competitive permit markets see Liski and Montero (2006), Cason et al. (2003), Atkinson and Tietenberg (2001), and Hahn (1984).
} 
by moving first the regulator is able to anticipate the effects that its choices of the subsidy rates and initial allocations have on the NPSs' subsequent abatement efforts (i.e., the NPSs' respective "response functions") and the per-unit price of abatement credits, $\rho$. In a subgame perfect equilibrium, the regulator correctly anticipates these response functions.

A subgame perfect equilibrium is solved through backward induction. We therefore begin our analysis with the second stage of the game, i.e., the WQT market. In a WQT market, NPS $j$ takes $\beta_{j}, \bar{a}_{j}$, and $\rho$ as given and chooses its abatement effort $a_{j}$ to minimize its cost of participating in the market, i.e., ${ }^{11}$

$$
\min _{a_{j}} \beta_{j} C_{j}\left(a_{j}\right)-\rho \tau_{j}\left[a_{j}-\bar{a}_{j}\right], \quad j=1,2
$$

where $C_{j}\left(a_{j}\right)$ is $j$ 's abatement cost function (increasing and convex in $a_{j}$ ) and $\tau_{j}$ is $j$ 's corresponding delivery ratio (i.e., transfer coefficient or conveyance loss). This problem results in "modified optimality conditions" for a competitive ambient permit market, where the modification is due to the appearance of the regulator's cost-share rates, $\beta_{j}$, directly in the NPSs' respective first-order conditions,

$$
\beta_{j}\left[\frac{\partial C_{j}}{\partial a_{j}}\right]=\rho \tau_{j}, \quad j=1,2
$$

The point source $P$ similarly chooses its abatement effort $a_{P}$ to minimize its cost of market participation,

$$
\min _{a_{P}} C_{P}\left(a_{P}\right)-\rho \tau_{P}\left[a_{P}-\bar{a}_{P}\right]
$$

resulting in corresponding optimality condition,

$$
\frac{\partial C_{P}}{\partial a_{P}}=\rho \tau_{P}
$$

Lastly, a market-clearing condition closes the solution,

$$
\bar{A}=\tau_{1} \bar{a}_{1}+\tau_{2} \bar{a}_{2}+\tau_{P} \bar{a}_{P} \leq \tau_{1} a_{1}+\tau_{2} a_{2}+\tau_{P} a_{P}
$$

where $\bar{A}$ is a receptor point's pre-determined aggregate abatement necessary to meet the target

\footnotetext{
${ }^{11}$ Parentheses denote a functional relationship, while square brackets indicate the application of a given operation on the included variables.
} 
delivered load, e.g., as specified in Total Maximum Daily Load (TMDL). ${ }^{12}$ Invoking the implicit function theorem, the NPSs' and point source's equilibrium abatement efforts, as well as the credit price, may be written as $a_{1} \equiv a_{1}\left(\beta_{1}, \beta_{2}\right), a_{2} \equiv a_{2}\left(\beta_{1}, \beta_{2}\right), a_{P} \equiv a_{P}\left(\beta_{1}, \beta_{2}\right), \rho \equiv \rho\left(\beta_{1}, \beta_{2}\right) .{ }^{13}$ Associated comparative statics for the system of equations (??) - (??) result in

$$
\frac{\partial a_{j}}{\partial \beta_{j}}<0, \frac{\partial \rho}{\partial \beta_{j}}>0, \frac{\partial a_{j}}{\partial \beta_{i}}>0, \quad i, j=1,2
$$

In other words, an NPS's abatement effort is decreasing in its own cost-share percentage and increasing in the cost-share percentage of the other NPS. This latter effect results indirectly from the positive relationship between an NPS's cost-share percentage and the equilibrium credit price.

In the first stage, the regulator chooses the NPSs' respective cost-share rates $\beta_{j}$ and initial allocations $\bar{a}_{j}$ to minimize its own cost-share obligation,

$$
\min _{\beta_{j}, \bar{a}_{j}}\left[1-\beta_{1}\right] C_{1}\left(a_{1}\left(\beta_{1}, \beta_{2}\right)\right)+\left[1-\beta_{2}\right] C_{2}\left(a_{2}\left(\beta_{1}, \beta_{2}\right)\right)
$$

subject to (??) and,

$$
\begin{gathered}
\tau_{1} \rho\left(\beta_{1}, \beta_{2}\right)\left[a_{1}\left(\beta_{1}, \beta_{2}\right)-\bar{a}_{1}\right]-\beta_{1} C_{1}\left(a_{1}\left(\beta_{1}, \beta_{2}\right)\right) \geq N B_{1}^{A l t} \\
\tau_{2} \rho\left(\beta_{1}, \beta_{2}\right)\left[a_{2}\left(\beta_{1}, \beta_{2}\right)-\bar{a}_{2}\right]-\beta_{2} C_{2}\left(a_{2}\left(\beta_{1}, \beta_{2}\right)\right) \geq N B_{2}^{A l t}
\end{gathered}
$$

where $N B_{j}^{A l t} \geq 0, j=1,2$, represents the net benefit of NPS $j$ 's next-best alternative. ${ }^{14}$ Equations (??) and (??) are the NPSs' respective participation constraints, i.e., the necessary profitability

\footnotetext{
${ }^{12}$ In models where the regulator is precluded from setting initial abatement allocations, e.g., $\bar{a}_{j}, j=1,2$, such as in the ambient penalty models of Segerson (1988) and Horan et al. (1998), monitoring and enforcement of NPS loads is essentially a non-issue. In contrast, the need for monitoring and enforcement in a WQT market would seem to naturally arise. However, as pointed out by Ross and Associates Environmental Consulting, Ltd. (2000), WQT is premised on the need for all parties to a trade (including the regulator itself) to agree on monitoring methods and measured outcomes before the trade can be transacted. Thus, in practical terms, monitoring and enforcement naturally occurs in WQT as a precursor to the trades themselves. We adopt this line of reasoning in our model by not explicitly endowing the regulator with the authority to levy penalties for cheating on the part of the NPSs or the point source. For a concise definition of TMDL see EPA (2011a).

${ }^{13}$ For expository purposes, the delivery ratios $\tau_{j}, j=1,2$, and $\tau_{P}$, and TMDL aggregate abatement level $\bar{A}$ are subsumed in these implicit functions. The initial allocations $\bar{a}_{j}, j=1,2$, and $\bar{a}_{P}$ affect neither market price $\rho$ nor abatement effort at an interior solution (only the agents' respective profitabilities).

${ }^{14} N B_{j}^{A l t}>0$ would need to hold in cases where NPS $j$ suffers a decrease in the profitability of its agricultural enterprise as a result of implementing a BMP, in which case revenues earned by participating as a seller in WQT would need to exceed the cost of BMP implementation.
} 
conditions that must be met before the NPSs will agree to participate in the WQT market with abatement-cost subsidization. Letting $\mu_{1}$ and $\mu_{2}$ represent the Lagrangian multipliers on constraints (??) and (??), respectively, and $\lambda$ the Lagrangian multiplier on equation constraint (??), the optimality conditions for the regulator's problem are (??), (??), (??), and

$$
\begin{gathered}
C_{1}(\cdot)-\left[1-\beta_{1}\right]\left[\frac{\partial C_{1}(\cdot)}{\partial a_{1}(\cdot)} \frac{\partial a_{1}(\cdot)}{\partial \beta_{1}}\right]=\left[1-\beta_{2}\right]\left[\frac{\partial C_{2}(\cdot)}{\partial a_{2}(\cdot)} \frac{\partial a_{2}(\cdot)}{\partial \beta_{1}}\right]-\mu_{1} \Omega_{11}-\mu_{2} \Omega_{12} \\
C_{2}(\cdot)-\left[1-\beta_{2}\right]\left[\frac{\partial C_{2}(\cdot)}{\partial a_{2}(\cdot)} \frac{\partial a_{2}(\cdot)}{\partial \beta_{2}}\right]=\left[1-\beta_{1}\right]\left[\frac{\partial C_{1}(\cdot)}{\partial a_{1}(\cdot)} \frac{\partial a_{1}(\cdot)}{\partial \beta_{2}}\right]-\mu_{1} \Omega_{21}-\mu_{2} \Omega_{22} \\
\mu_{1}=\mu_{2}
\end{gathered}
$$

and

$$
\begin{gathered}
\Omega_{11}=C_{1}(\cdot)+\beta_{1}\left[\frac{\partial C_{1}(\cdot)}{\partial a_{1}(\cdot)} \frac{\partial a_{1}(\cdot)}{\partial \beta_{1}}\right]-\tau_{1}\left[\frac{\partial \rho(\cdot)}{\partial \beta_{1}}\right]\left[a_{1}(\cdot)-\bar{a}_{1}\right]-\rho(\cdot) \tau_{1}\left[\frac{\partial a_{1}(\cdot)}{\partial \beta_{1}}\right] \\
\Omega_{12}=\beta_{2}\left[\frac{\partial C_{2}(\cdot)}{\partial a_{2}(\cdot)} \frac{\partial a_{2}(\cdot)}{\partial \beta_{1}}\right]-\tau_{2}\left[\frac{\partial \rho(\cdot)}{\partial \beta_{1}}\right]\left[a_{2}(\cdot)-\bar{a}_{2}\right]-\rho(\cdot) \tau_{2}\left[\frac{\partial a_{2}(\cdot)}{\partial \beta_{1}}\right] \\
\Omega_{21}=\beta_{1}\left[\frac{\partial C_{1}(\cdot)}{\partial a_{1}(\cdot)} \frac{\partial a_{1}(\cdot)}{\partial \beta_{2}}\right]-\tau_{1}\left[\frac{\partial \rho(\cdot)}{\partial \beta_{2}}\right]\left[a_{1}(\cdot)-\bar{a}_{1}\right]-\rho(\cdot) \tau_{1}\left[\frac{\partial a_{1}(\cdot)}{\partial \beta_{2}}\right] \\
\Omega_{22}=C_{2}(\cdot)+\beta_{2}\left[\frac{\partial C_{2}(\cdot)}{\partial a_{2}(\cdot)} \frac{\partial a_{2}(\cdot)}{\partial \beta_{2}}\right]-\tau_{2}\left[\frac{\partial \rho(\cdot)}{\partial \beta_{2}}\right]\left[a_{2}(\cdot)-\bar{a}_{2}\right]-\rho(\cdot) \tau_{2}\left[\frac{\partial a_{2}(\cdot)}{\partial \beta_{2}}\right] .
\end{gathered}
$$

One obvious corner solution to this problem would be to (1) provide zero subsidies to the NPSs, i.e., set $\beta_{1}=\beta_{2}=1$, (2) shift the entire initial abatement allocation to the point source, i.e., set $\bar{a}_{1}=\bar{a}_{2}=0$ and $\bar{a}_{P}=\bar{A} / \tau_{P}$, and, (3) given sufficient abatement-cost differences between the NPSs and the point source, rely on the water quality market to generate profits for the NPSs. This would result in zero cost to the regulator. However, in the case of $N B_{j}^{A l t}>0$ for some $j$, relying solely on WQT to generate the requisite profit for unsubsidized NPSs may not ensure satisfaction of the NPS participation constraints, e.g., if NPS $j$ 's profit generated through trading is not larger than $N B_{j}^{A l t}$. More importantly, because we are explicitly investigating the relationship between WQT and the subsidization of NPS abatement costs, we confine our analysis to interior solutions.

Equations (??) and (??) solve for the regulator's benchmark cost-share rates $\beta_{1}$ and $\beta_{2}$, respectively, under full-information. The left-hand sides of these two equations represent the marginal benefits (in terms of reduced cost-share obligations for the regulator) associated with incremental 
increases in the respective $\beta_{j} \mathrm{~s}$, while the right-hand sides represent the associated marginal costs. For example, in equation (??) marginal benefits accrue to the regulator through a reduction in NPS 1's abatement cost, and thus in the attendant subsidy cost incurred by the regulator. In specific, marginal benefits occur through (1) a reduction in NPS 1's abatement effort (and thus its corresponding abatement cost, which in turn reduces the cost shared by the regulator), i.e., the term $\left[1-\beta_{1}\right]\left[\frac{\partial C_{1}(\cdot)}{\partial a_{1}(\cdot)} \frac{\partial a_{1}(\cdot)}{\partial \beta_{1}}\right]<0$, and $(2)$ a direct reduction in the regulator's share of NPS 1's abatement cost (i.e., the term $\left.C_{1}(\cdot)>0\right) .{ }^{15}$ Marginal costs consist of (1) a "cross-effect" increase in the abatement effort (and corresponding abatement cost) of NPS 2 (i.e., the term $\left[1-\beta_{2}\right]\left[\frac{\partial C_{2}(\cdot)}{\partial a_{2}(\cdot)} \frac{\partial a_{2}(\cdot)}{\partial \beta_{1}}\right]>0$ ) and (2) the added net costs associated with changes in the profitabilities of NPSs 1 and 2, and thus the ability of the regulator to satisfy participation constraints (??) and (??), i.e. the terms $\mu_{1} \Omega_{11}$ and $\mu_{2} \Omega_{12}$. Finally, equation (??) results from the regulator's choice of the initial allocations $\bar{a}_{j}, j=1,2$ and reflects the fact that the regulator values the participation of each NPS equally on the margin. Solving equations (??) - (??) simultaneously establishes the first-best benchmark, characterized by the value set $\left(\beta_{1}^{*}, \beta_{2}^{*}, a_{j}^{*}, \bar{a}_{j}^{*}, a_{P}^{*}, \bar{a}_{P}^{*}, \rho^{*}, \mu_{1}^{*}, \mu_{2}^{*}, \lambda^{*}\right), j=1,2$.

\subsection{The Second-Best Problem}

As alluded to earlier, uncertainty about the NPSs' abatement costs limits the regulator's ability to implement the first-best benchmark derived in Section 2.1. An additional type of uncertainty pertains to the diffuse nature of NPS emissions and the whims of nature, which can affect the ultimate effectiveness of any given level of abatement effort implemented by an NPS. In other words, abatement effort at the source does not necessarily translate into a certain measured reduction in concentration at the receptor point, due to environmental factors such as seasonal or weather-related variations as well as the general limitations of hydrological and topographical data. To overcome this type of uncertainty we have assumed that the regulator (1) selects the receptor point's target concentration $\bar{A}$ with certainty (in accordance with a TMDL, as mentioned previously), (2) likewise sets the NPSs' respective delivery ratios, $\tau_{j}, j=1,2$, and $\tau_{P}$ in accordance with accurate (albeit probabilistic) scientific information, and (3) as importantly, communicates this information (along

\footnotetext{
${ }^{15}$ There is a scaling issue associated with this direct-reduction effect - with respect to the first-order conditions for both $\beta_{1}$ and $\beta_{2}$ - due to the fact that the $\beta_{j}$ s are measured in percentage terms and the abatement costs are measured in (potentially large) dollar values. This issue is addressed in Section 3 by scaling $C_{j}(\cdot), j=1,2$ in conditions (??) and (??) each by a factor of 0.01 in order to reduce their values to a scale commensurate with that of the respective $\beta_{j} \mathrm{~s}$.
} 
with $\left.\bar{a}_{j}, j=1,2\right)$ to the NPSs. Recent research suggests that estimation of seasonal, field-level nutrient loadings and delivery ratios through the application of sophisticated hydrologic models can provide "reasonable assurance" that TMDL standards can be met through WQT (Caplan, et al., 2009; Horan, et al., 2002a and 2002b) and thus overcome the type of environmental uncertainty explicitly modeled in Malik, et al. (1993) and Shortle and Horan (2001). In this way, the target concentration and delivery ratios play similar roles as concentration standards in the Segerson (1988) and Horan, et al. (1998) linear ambient tax models, and the communication of tax schedules in the Shortle and Dunn (1986) management-practices models.

The uncertainty left unresolved in this paper pertains to an NPS's ability to misrepresent itself, i.e., to "hide" information from the regulator with respect to whether it is able to abate at high or low cost. For instance, if a low-cost NPS believes the regulator's cost-share rule is monotone in abatement costs (i.e., NPSs with higher abatement cost functions tend to receive higher subsidy rates) the NPS may have incentive to misrepresent itself as high cost in order to receive a lower $\beta_{j}$ and $\bar{a}_{j}{ }^{16}$ To capture the potential effect of this hidden information, we introduce standard probability parameters (i.e., odds ratios) $\pi_{j}>0, j=1,2$, which represents the regulator's estimate of the (relative) likelihood that NPS $j$ is indeed providing the regulator with its true abatement cost information; information the regulator then uses to set NPS $j$ 's $\beta_{j}$ and $\bar{a}_{j}$.

To further motivate our analysis, we assume that NPS 1 is a low-cost producer of abatement and NPS 2 is a high-cost producer, i.e., $C_{2}(a)>C_{1}(a)$ for any common abatement level $a$. Further, the difference in costs abides by the single-crossing property, i.e.,

$$
\frac{\partial C_{2}(a)}{\partial a}>\frac{\partial C_{1}(a)}{\partial a}
$$

for any common $a$. Thus, not only is NPS 2's abatement cost everywhere larger than NPS 1's, but it also increases at a faster rate. In addition to being a standard assumption in hidden-information models of the type developed here (see Varian, 1992), the single-crossing property is reflective of the underlying presumption - first mentioned in Section 1 - of divergence between the NPSs' abatement costs.

\footnotetext{
${ }^{16}$ For simplicity we assume that each NPS has only two possible abatement cost schedules - its own and that of the other NPS. Assuming the NPSs have wider cost spaces would unnecessarily complicate the ensuing analysis without adding much by way of insight. This is a common approach to modeling the hidden information problem. See Varian (1992) and Hart and Holmstrom (1987) for further discussion.
} 
As a result of the regulator's choices in the first stage, the NPSs are subsequently induced to portray themselves truthfully. Truthful revelation occurs as a result of the regulator satisfying both the participation and incentive-compatibility constraints in its first-stage problem (see below). Thus, NPS $j$ 's (as well as point source $P$ 's) cost minimization problem in the second stage of its game with the regulator is identical to that described in Section 2.1. The resulting optimality conditions are (??) - (??), which again characterize the second-stage WQT market equilibrium.

In the first stage of the game, the regulator again chooses the NPSs' respective cost-share rates $\beta_{j}$ and initial allocations $\bar{a}_{j}$ to minimize its cost-share obligation, ${ }^{17}$

$$
\min _{\beta_{j}, \bar{a}_{j}} \pi_{1}\left[1-\beta_{1}\right] C_{1}\left(a_{1}\left(\beta_{1}, \beta_{2}\right)\right)+\pi_{2}\left[1-\beta_{2}\right] C_{2}\left(a_{2}\left(\beta_{1}, \beta_{2}\right)\right)
$$

subject to (??) - (??), (??), (??), and,

$$
\begin{array}{r}
\tau_{1} \rho\left(\beta_{1}, \beta_{2}\right)\left[a_{1}\left(\beta_{1}, \beta_{2}\right)-\bar{a}_{1}\right]-\beta_{1} C_{1}\left(a_{1}\left(\beta_{1}, \beta_{2}\right)\right) \geq \\
\tau_{1} \rho\left(\beta_{1}, \beta_{2}\right)\left[a_{2}\left(\beta_{1}, \beta_{2}\right)-\bar{a}_{2}\right]-\beta_{2} C_{1}\left(a_{2}\left(\beta_{1}, \beta_{2}\right)\right) \\
\tau_{2} \rho\left(\beta_{1}, \beta_{2}\right)\left[a_{2}\left(\beta_{1}, \beta_{2}\right)-\bar{a}_{2}\right]-\beta_{2} C_{2}\left(a_{2}\left(\beta_{1}, \beta_{2}\right)\right) \geq \\
\tau_{2} \rho\left(\beta_{1}, \beta_{2}\right)\left[a_{1}\left(\beta_{1}, \beta_{2}\right)-\bar{a}_{1}\right]-\beta_{1} C_{2}\left(a_{1}\left(\beta_{1}, \beta_{2}\right)\right)
\end{array}
$$

where we henceforth assume $N B_{1}^{A l t}=N B_{2}^{A l t} \geq 0 .{ }^{18}$ Equations (??) and (??) are standard selfselection (or incentive-compatibility) constraints. For example, constraint (??) states that costshares $\beta_{1}$ and $\beta_{2}$ are chosen such that the profit NPS 1 obtains from adopting its true (low-cost) level of abatement effort is never less than the profit it would obtain by adopting NPS 2's true (high-cost) abatement level (which NPS 1 would conceivably do if it thinks the added benefits associated with obtaining from the regulator $\beta_{2}$ (rather than $\beta_{1}$ ) and $\bar{a}_{2}$ (rather than $\bar{a}_{1}$ ) outweigh the added costs associated with the higher-cost level of abatement). Constraint (??) does likewise

\footnotetext{
${ }^{17}$ We follow Varian (1992) and Mas-Colell et al. (1995) by retaining the odds ratios in the regulator's objective function. Since we ultimately assume the regulator is able to simultaneously satisfy both the participation and incentive-compatibility constraints (introduced below), the odds ratios essentially become superfluous to this problem. In other words, by the Revelation Principle if incentive compatibility constraints are satisfied, truth-telling is the dominant strategy and thus there is no randomness remaining in the regulator's objective function. See Baron and Myerson (1982) for a discussion of the Revelation Principle.

${ }^{18}$ The assumption of equally valued next-best alternatives simplifies the ensuing analysis without qualitatively changing the results in this section, as well as the numerical results in Section 3.
} 
for NPS 2. ${ }^{19}$

To begin, we compare constraints (??) and (??). Using (??), and the fact that NPS 1(2) is low(high) cost, we see that when

$$
\begin{gathered}
\tau_{1} \rho\left(\beta_{1}, \beta_{2}\right)\left[a_{2}\left(\beta_{1}, \beta_{2}\right)-\bar{a}_{2}\right]-\beta_{2} C_{1}\left(a_{2}\left(\beta_{1}, \beta_{2}\right)\right) \geq \\
\tau_{2} \rho\left(\beta_{1}, \beta_{2}\right)\left[a_{2}\left(\beta_{1}, \beta_{2}\right)-\bar{a}_{2}\right]-\beta_{2} C_{2}\left(a_{2}\left(\beta_{1}, \beta_{2}\right)\right) \geq N B_{2}^{A l t}=N B_{1}^{A l t} \geq 0,
\end{gathered}
$$

e.g., when $\tau_{1} \geq \tau_{2}$, constraint (??) does not bind, implying that constraint (??) does. ${ }^{20}$ By contrast, when conditions are such that (??) does not bind, then as long as constraint (??) binds and $\tau_{2}>\tau_{1}$ by a sufficient amount, constraint (??) will bind as well.

We can compare constraints (??) and (??) in a similar manner. Again assuming (??) binds, it can be substituted to (??) resulting in,

$$
\frac{\beta_{2} \tau_{2} C_{1}\left(a_{2}\left(\beta_{1}, \beta_{2}\right)\right)}{\tau_{1}}-\frac{\beta_{1} \tau_{2} C_{1}\left(a_{1}\left(\beta_{1}, \beta_{2}\right)\right)}{\tau_{1}} \geq \beta_{2} C_{2}\left(a_{2}\left(\beta_{1}, \beta_{2}\right)\right)-\beta_{1} C_{2}\left(a_{1}\left(\beta_{1}, \beta_{2}\right)\right)
$$

By condition (??), and for (1) inequality $a_{1}(\cdot)>a_{2}(\cdot)$ (which, for small enough differences between $\tau_{1}$ and $\tau_{2}$, is a natural outcome given $C_{2}(a)>C_{1}(a)$ for any $a$ ) and (2) similarly small enough differences between $\beta_{1}$ and $\beta_{2}$, constraint (??) does not bind (implying that (??) binds). This particular result - where (??) and (??) are the binding constraints - is common in the hiddeninformation literature (e.g., Varian, 1992; Mas-Colell, et al., 1995). Here, it indicates that the regulator chooses $\beta_{1}$ and $\beta_{2}$ such that (1) the high-cost NPS just obtains its reservation profit (which nonetheless satisfies its incentive-compatibility constraint slack), and (2) the low-cost NPS receives more than its reservation profit (but still a profit level that just satisfies its incentivecompatibility constraint). For this particular case, therefore, we obtain a system of equations that includes the regulator's first-stage optimality conditions, equations (??) - (??), binding constraints

\footnotetext{
${ }^{19}$ Note that for this problem we do not have $\rho\left(\beta_{2}, \beta_{2}\right)$ and $a_{2}\left(\beta_{2}, \beta_{2}\right)$ on the right-hand side of (??) because these reaction functions must nevertheless account for the responses of $\rho$ and $a_{2}$ in the WQT market to the regulator's previous choices of $\beta_{1}$ and $\beta_{2}$ in the first stage of the game. Likewise with respect to not having $\rho\left(\beta_{1}, \beta_{1}\right.$ and $a_{1}\left(\beta_{1}, \beta_{1}\right)$ on the right-hand side of (??).

${ }^{20}$ Note that $\tau_{1} \geq \tau_{2}$ is sufficient but not necessary for the above inequality to hold.
} 
(??) and (??), and ${ }^{21}$

$$
\begin{gathered}
\pi_{1} C_{1}(\cdot)-\pi_{1}\left[1-\beta_{1}\right]\left[\frac{\partial C_{1}(\cdot)}{\partial a_{1}(\cdot)} \frac{\partial a_{1}(\cdot)}{\partial \beta_{1}}\right]=\pi_{2}\left[1-\beta_{2}\right]\left[\frac{\partial C_{2}(\cdot)}{\partial a_{2}(\cdot)} \frac{\partial a_{2}(\cdot)}{\partial \beta_{1}}\right]-\mu_{1} \Psi_{11}-\mu_{2} \Psi_{12} \\
\pi_{2} C_{2}(\cdot)-\pi_{2}\left[1-\beta_{2}\right]\left[\frac{\partial C_{2}(\cdot)}{\partial a_{2}(\cdot)} \frac{\partial a_{2}(\cdot)}{\partial \beta_{2}}\right]=\pi_{1}\left[1-\beta_{1}\right]\left[\frac{\partial C_{1}(\cdot)}{\partial a_{1}(\cdot)} \frac{\partial a_{1}(\cdot)}{\partial \beta_{2}}\right]-\mu_{1} \Psi_{21}-\mu_{2} \Psi_{22} \\
\mu_{2}=\frac{\tau_{2} \mu_{1}}{\tau_{1}+\tau_{2}}
\end{gathered}
$$

where $\mu_{1}$ and $\mu_{2}$ now represent the multipliers on constraints (??) and (??), respectively, and

$$
\begin{gathered}
\Psi_{11}=-\beta_{2}\left[\frac{\partial C_{2}(\cdot)}{\partial a_{2}(\cdot)} \frac{\partial a_{2}(\cdot)}{\partial \beta_{1}}\right]+\tau_{2}\left[\frac{\partial \rho(\cdot)}{\partial \beta_{1}}\right]\left[a_{2}(\cdot)-\bar{a}_{2}\right]+\rho(\cdot) \tau_{2}\left[\frac{\partial a_{2}(\cdot)}{\partial \beta_{1}}\right] \\
\Psi_{12}=-C_{1}(\cdot)-\beta_{1}\left[\frac{\partial C_{1}(\cdot)}{\partial a_{1}(\cdot)} \frac{\partial a_{1}(\cdot)}{\partial \beta_{1}}\right]-\tau_{1}\left[\frac{\partial \rho(\cdot)}{\partial \beta_{1}}\right]\left[a_{2}(\cdot)-\bar{a}_{2}\right]+\rho(\cdot) \tau_{1}\left[\frac{\partial a_{1}(\cdot)}{\partial \beta_{1}}\right] \\
+\tau_{1}\left[\frac{\partial \rho(\cdot)}{\partial \beta_{1}}\right]\left[a_{1}(\cdot)-\bar{a}_{1}\right]-\rho(\cdot) \tau_{1}\left[\frac{\partial a_{2}(\cdot)}{\partial \beta_{1}}\right]+\beta_{2}\left[\frac{\partial C_{1}(\cdot)}{\partial a_{2}(\cdot)} \frac{\partial a_{2}(\cdot)}{\partial \beta_{1}}\right] \\
\Psi_{21}=-\beta_{2}\left[\frac{\partial C_{2}(\cdot)}{\partial a_{2}(\cdot)} \frac{\partial a_{2}(\cdot)}{\partial \beta_{2}}\right]+\tau_{2}\left[\frac{\partial \rho(\cdot)}{\partial \beta_{2}}\right]\left[a_{2}(\cdot)-\bar{a}_{2}\right]+\rho(\cdot) \tau_{2}\left[\frac{\partial a_{2}(\cdot)}{\partial \beta_{2}}\right]-s C_{2}(\cdot) \\
\Psi_{22}=C_{1}(\cdot)-\beta_{1}\left[\frac{\partial C_{1}(\cdot)}{\partial a_{1}(\cdot)} \frac{\partial a_{1}(\cdot)}{\partial \beta_{2}}\right]-\tau_{1}\left[\frac{\partial \rho(\cdot)}{\partial \beta_{2}}\right]\left[a_{2}(\cdot)-\bar{a}_{2}\right]+\rho(\cdot) \tau_{1}\left[\frac{\partial a_{1}(\cdot)}{\partial \beta_{2}}\right] \\
+\tau_{1}\left[\frac{\partial \rho(\cdot)}{\partial \beta_{2}}\right]\left[a_{1}(\cdot)-\bar{a}_{1}\right]-\rho(\cdot) \tau_{1}\left[\frac{\partial a_{2}(\cdot)}{\partial \beta_{2}}\right]+\beta_{2}\left[\frac{\partial C_{1}(\cdot)}{\partial a_{2}(\cdot)} \frac{\partial a_{2}(\cdot)}{\partial \beta_{2}}\right]
\end{gathered}
$$

Solving equations (??) - (??), binding (??) and (??), and (??) - (??) simultaneously establishes this particular second-best outcome, characterized by the value set $\left(\beta_{1}^{\prime}, \beta_{2}^{\prime}, a_{j}^{\prime}, \bar{a}_{j}^{\prime}, a_{P}^{\prime}, \bar{a}_{P}^{\prime}, \rho^{\prime}, \mu_{1}^{\prime}, \mu_{2}^{\prime}, \lambda^{\prime}\right), j=$ 1,2 , where $\lambda^{\prime}$ is the multiplier on constraint (??). Note that (??) is consistent with the low-cost NPS receiving an informational rent from the regulator, since the added cost to the regulator of allowing high-cost NPS 2 to exceed its reservation profit level (i.e., $\mu_{1}$ ) is larger than the added cost of allowing low-cost NPS 1 to exceed its incentive-compatible profit level (i.e., $\left.\mu_{2}\right)$.

To see this particular result more clearly, we now turn to numerical simulations of the basic model. As shown in the next section, these simulations are based on simple functional forms for the NPSs' and point source's abatement cost functions. The numerical simulations also consider cases

\footnotetext{
${ }^{21}$ As with conditions (??) and (??) in Section 2.1, the direction-reduction effects in conditions (??) and (??), i.e., the $\pi_{1} C_{1}$ and $\pi_{2} C_{2}$ terms, are ultimately scaled by a factor of 0.01 for the numerical simulations in Section 3 .
} 
where other sets of constraints are binding besides (??) and (??). Thus, we are able to distinguish a unique second-best benchmark for the numerical model.

\section{$3 \quad$ Numerical Simulations}

In order to simulate possible equilibrium outcomes under full and incomplete information, we assume the following abatement cost functions for NPSs 1 and 2 and point source $P$, respectively, ${ }^{22}$

$$
\begin{gathered}
C_{1}\left(a_{1}\right)=\frac{a_{1}^{2}}{2} \\
C_{2}\left(a_{2}\right)=\frac{2 a_{2}^{2}}{3} \\
C_{P}\left(a_{P}\right)=a_{P}^{2}
\end{gathered}
$$

Thus, NPS 2(1) is high(low) cost and condition (??) is duly satisfied. Table 1 contains the various parameter values for our initial and alternative simulation exercises. Note that for the initial simulation we assume that NPS 1 is located "downstream" from NPS 2 vis-a-vis the receptor point, i.e., $\tau_{1}=1>\tau_{2}=0.95$, and that the relative likelihoods of each NPS choosing to abate according to their true abatement costs are each equal to 2, i.e., $\pi_{1}=\pi_{2}=2 .{ }^{23}$ Results for the first- and second-best scenarios for this initial simulation are presented in Table 2.

For the first-best benchmark, note from Table 2 that NPS 2 receives a slightly lower subsidy in percentage terms, i.e., it shares $51 \%$ of its abatement cost with the regulator while NPS 1 shares $49 \%$ of its cost. These cost-share rates are generally higher than the typical EQIP cost-share rates reported by the NRCS, which range between 25 and 40 percent (i.e., between 60 and 75 percent when defining the rate as percentage of cost incurred by the NRCS)(NRCS, 2011a and 2011b). In contrast, NPS 2 receives a lower initial abatement allocation (24 units vs. NPS 1's 37 units). As a result of WQT, NPS 1 abates more than NPS 2 (79 units vs. 54 units). The excess of abatement

\footnotetext{
${ }^{22}$ GAMS IDE version 2.0.20.0 is used to generate the simulations. Copies of the input code are available upon request from the author.

${ }^{23}$ Recall from Section 2.2 that unambiguous analytical results for the second-best solution depend on sufficiently small differences between $\tau_{1}$ and $\tau_{2}$. We nevertheless perform sensitivity analysis on the values of $\tau_{j}$ in order to test the stability of our simulations. Also, we assume that the regulator has access to enough information about the NPSs' respective abatement cost functions to be relatively confident about which NPS is which, as evidenced by the relatively large values assumed for $\pi_{1}$ and $\pi_{2}$, e.g., the regulator believes that NPS 1 is two times as likely to identify itself as NPS 1 than is NPS 2 and vice-versa for NPS 2 .
} 
over initial allocation obtained by each respective NPS is then sold to point source $P$, so that $P$ can effectively meet its abatement allocation, i.e., $(89.87-19.46)=(79.00-36.93)+(54.26-24.42)$. The regulator effectively ensures that each NPS's surplus (i.e., profit) from participating in WQT equals the value of its next best alternative (i.e., participation constraints (??) and (??) bind). However, point source $P$ obtains negative profit (driven by its relatively large initial abatement allocation and the fact that it does not receive a subsidy from the regulator). ${ }^{24}$ The total cost of the subsidy program for the regulator amounts to approximately $\$ 2,540$.

The third column of Table 2 contains the results for the second-best scenario. In reacting to the uncertainties associated with NPS costs and abatement effectiveness, the regulator maintains its subsidization rate for NPS 1 and increases NPS 2's (NPS 2's cost-share falls from 51\% to 48\%). In addition, the regulator shifts more of the initial abatement allocation toward point source $P$ (from 90 units in the ex post outcome to 98 units in the ex ante outcome). As a result, the initial allocation to NPS 1 is reduced by more than the allocation is increased for NPS 2 (from 37 to 28 units for NPS 1 and from 24 to 26 units for NPS 2). ${ }^{25}$

Further, the pattern of trade in the permit market is altered. NPS 1(2) now abates less(more), and the point source in turn abates slightly less itself. Although the equilibrium permit price is slightly lower, the negative profit incurred by the point source is now larger (from approximately $-\$ 3,100$ to $-\$ 3,400)$. Although NPS 2's profit is constrained to equal the value of its next-best alternative, NPS 1, being the low-cost NPS, is able to extract a rent from the regulator worth approximately $\$ 316$. Finally, the regulator's total cost rises, from approximately $\$ 2,540$ in the second-best scenario to $\$ 2,630$ in the first-best scenario, indicating an "informational cost" to the regulator of approximately $\$ 90$, or only $4 \%$.

As indicated in Table 1, we test the stability of the simulation model by examining an alternative scenario where the NPSs' respective delivery ratios are reversed, i.e., NPS 2 is now located downstream from NPS 1. Table 3 reports the results. Due to the ambiguity associated with the second-best outcome for the case of $\tau_{2}>\tau_{1}$ (as discussed in Section 2.2), we have solved the regulator's first-stage problem for each of the three combinations of constraints that the regulator could

\footnotetext{
${ }^{24}$ Note that the regulator is precluded from simply assigning the entire initial abatement allocation $\bar{A}$ to point source $P$ because of the binding participation constraints for both NPSs. This preclusion is also evident in the second-best scenario discussed below, where only NPS 2's participation constraint binds.

${ }^{25}$ Thus, the regulator in effect compensates NPS 1 for not having received a decrease in its cost share with a larger decrease in its initial abatement allocation.
} 
conceivably face, and have then selected the solution corresponding to the lowest total abatement cost. The three constraint combinations are represented by equations $\{(? ?),(? ?)\},\{(? ?),(? ?)\}$, and $\{(? ?),(? ?)\}$. As demonstrated in Section 2.2, the constraints included in combinations $\{(? ?),(? ?)\}$ and $\{(? ?),(? ?)\}$ cannot hold simultaneously. In addition, the constraint combination $\{(? ?),(? ?)\}$ is incompatible with the regulator's choices of $\bar{a}_{j}, j=1,2$ (a proof of this result is available from the authors upon request). In our case, constraint set $\{(? ?),(? ?)\}$ results in the lowest total cost of regulation for the regulator and is therefore considered the set of binding constraints for the second-best solution to this problem.

Comparing the first-best results in Table 3 with those in Table 2, we see that the resulting equilibrium changes only slightly in response to the changes in the delivery ratios. In relative terms, all of the results obtained for the initial first-best equilibrium are retained in this alternative equilibrium, e.g., NPS 2 receives a slightly lower subsidy in percentage terms than NPS 1, as well as a lower initial abatement allocation.

However, with respect to the second-best equilibrium, the changes are more pronounced. Although NPS 1's cost-share rate stays constant at 48\%, NPS 2's increases to $52 \%$ (or, alternatively stated, its subsidy rate falls from $57 \%$ to $48 \%$ ). In concert with these rates, NPS 1's(2's) abatement allocation rises(falls) from 34(27) to 36(26) units. Thus, although NPS 2's loadings are now relatively more potent than NPS 1's (due to $\tau_{2}>\tau_{1}$ ), and thus, all else equal, it is more cost effective to encourage more abatement from NPS 2, the regulator nevertheless balances a lower abatement allocation for NPS 2 with a lower subsidy rate. By eliminating NPS 1's rent payment, which is a consequence of constraint (??) now binding (rather than (??)), the regulator is able to reduce its total cost to $\$ 2,591$, which is now only slightly larger than the first-best benchmark of $\$ 2,573$.

\section{Summary}

This paper has examined how a regulatory authority might consider subsidizing the participation of nonpoint sources (through a cost-sharing arrangement) in the surplus-generating economic activity of water quality trading. Toward this end, the subgame-perfect equilibrium concept is adopted to model the "multilateral contracting" relationship between the regulatory authority and the nonpoint sources when the regulator has incomplete information about the nonpoint sources' respective 
abatement costs. We derive analytical expressions for first-best allocations in the presence of regulator uncertainty regarding these costs. The extent to which the first-best allocation is constrained by asymmetric information concerning the nonpoint sources' costs is also demonstrated analytically. In numerical analysis we find that the regulator reacts to its problem of incomplete information by increasing its cost-share ratios and, in some cases, providing additional rent to the low-cost nonpoint source. It also shifts more of the required abatement to the point source, for which there is no asymmetric information problem. As a result, the overall cost of regulation increases relative to the first-best benchmark.

Our results suggest that a cost-sharing arrangement in the presence of incomplete information about an nonpoint sources' abatement costs can successfully be coupled with the development of a market within which the nonpoint sources are able to generate surplus through trading abatement credits with one another and with point sources. In cases where the nonpoint sources can voluntarily decide whether to enter the cost-sharing arrangement with the regulator, such as for the control of nonpoint pollution, the development of such a market may be a strong enough inducement for the nonpoint sources to enter the cost-sharing arrangement in the first place. 


\section{References}

Atkinson, S.E. and T. Tietenberg (2001) "Market failure in incentive-based regulation: The case of emissions trading." In Tietenberg, T. (Ed.) Emissions trading programs, volume 2, theory and design. Aldershot, U.K.: Ashgate.

Baron, D.P. and R.B. Myerson (1982) "Regulating a monopolist with unknown costs." Econometrica, 50(4), 911-930.

Bolton, P. and M. Dewatripont (2005) Contract theory. MIT Press, Cambridge, MA.

Breetz, H.L. and K. Fisher-Vanden (2007) "Does cost-share replicate water quality trading projects? Implications for a possible partnership." Review of Agricultural Economics, 29(2), 201-215.

Breetz, H.L., K. Fisher-Vanden, L. Garzon, H. Jacobs, K. Kroetz, and R. Terry (2004) "Water quality trading and offsets initiatives in the U.S.: a comprehensive survey." Unpublished manuscript, Dartmouth College, Hanover, New Hampshire.

Breetz, H.L., K. Fisher-Vanden, H. Jacobs, and C. Schary (2005) "Trust and communication: mechanisms for increasing farmers' participation in water quality trading." Land Economics, 81(2), 170-190.

Caplan, A.J., B.T. Neilson, and M. Baker (2009) "Going with the flow: water quality trading in an integrated hydrologic modelling framework." Economic Research Institute Working Paper 2009-05, Utah State University.

Caplan, A.J., R.C. Cornes, and E.C.D. Silva (2000) "Pure public goods and income redistribution in a federation with decentralized leadership and imperfect labor mobility." Journal of Public Economics, 77(2), 265-284.

Cason, T.N., L. Gangadharan, and C. Duke (2003) "Market power in tradable emission markets: A laboratory testbed for emission trading in Port Phillip Bay, Victoria." Ecological Economics, 46(3), 469-491.

Environomics (1999) "A summary of U.S. effluent trading and offset projects." Report prepared for the U.S. Environmental Protection Agency, Office of Water Quality. 
Farrow, R.S., M.T. Schultz, P. Celikkol, and G.L. Van Houtven (2005) "Pollution trading in water quality limited areas: use of benefits assessment and cost-effective trading ratios." Land Economics, 81(2), 191-205.

Freeman, A.M. (2002) "Environmental policy since earth day I: what have we gained?" Journal of Environmental Perspectives, 16(1), 125-146.

Hahn, R.W. (1984) "Market power and transferable property rights." Quarterly Journal of Economics, 99, 753-765.

Hall, L. and E. Raffini (2005) "Water quality trading: where do we go from here?" Natural Resources and Environment, 20(2), 38-42.

Hart, O. and B. Holmstrom (1987) "The theory of contracts." In T. Bewley (Ed.), Advances in Economic Theory. Cambridge: Cambridge University Press.

Hoag, D.L. and J.S. Hughes-Popp (1997) "Theory and practice of pollution credit trading in water quality management." Review of Agricultural Economics, 19(2), 252-262.

Horan, R.D., D.G. Abler, J.S. Shortle, J. Carmichael (2002a) "Cost effective point-nonpoint trading: an application to the Susquehanna river basin." Journal of the American Water Resources Association, 38(2), 467-477.

Horan, R.D. (2001) "Differences in social and public risk perceptions and conflicting impacts on point/nonpoint trading ratios." American Journal of Agricultural Economics, 83, 934-941.

Horan, R.D., J.S. Shortle, and D.G. Abler (1998) "Ambient taxes when polluters have multiple choices." Journal of Environmental Economics and Management, 36, 186-199.

Horan, R.D., J.S. Shortle, and D.G. Abler (2002b) "Point-nonpoint nutrient trading in the Susquehanna river basin." Water Resources Research, 38(5), 1050-1062.

Horan, R.D., J.S. Shortle, and D.G. Abler (2004) "The coordination and design of point-nonpoint trading programs and agri-environmental policies." Agricultural and Resource Economics Review, 33(1), 61-78. 
Isik, H. and B. Sohngen (2003) "Performance-based voluntary group contracts for nonpoint source pollution." Paper prepared for presentation at the American Agricultural Economics Association Annual Meeting, Montreal, Canada, July 27-30, 2003.

King, D.M. (2005) "Crunch time for water quality trading." Choices, 20(1), 71-75.

King, D.M. and P.J. Kuch (2003) "Will nutrient credit trading ever work? An assessment of supply and demand problems and institutional obstacles." Environmental Law Reporter, 33, 10362-10368.

Kramer, J. (2003) Lessons from the trading pilots: applications for Wisconsin water quality trading policy. Resource Strategies, Madison, Wisconsin.

Laffont, J-J and J. Tirole (1993) A theory of incentives in procurement and regulation. MIT Press, Cambridge, MA.

Liski, M. and J.-P. Montero (2006) "On pollution permit banking and market power." Journal of $\underline{\text { Regulatory Economics, 29(3), } 283302 .}$

Malik, A., D. Letson, and S. Crutchfield (1993) "Point/nonpoint source trading of pollution abatement: choosing the right trading ratio." American Journal of Agricultural Economics 75, 959-967.

Mas-Colell, A., M.D. Whinston, and J.R. Green (1995) Microeconomic theory. Oxford University Press, New York, NY.

National Association of Conservation Districts (NACD) (2003) Report of the conservation $\underline{\text { innovations task force (CITF), appendix III - water quality trading - nonpoint credit bank }}$

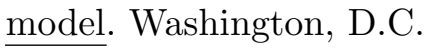

National Resource Conservation Service (NRCS) (2011a). Retrieved from the internet on September 26, 2011 at http://www.nrcs.usda.gov/Programs/eqip/.

National Resource Conservation Service (NRCS) (2011b). Retrieved from the internet on September 26, 2011 at http://www.il.nrcs.usda.gov/programs/eqip/cspractices.html.

Pushkarskaya, H. (2003) "Nonpoint source water pollution control: incentives theory approach." PhD dissertation, The Ohio State University. 
Ribaudo, M. (2001) "Non-point source pollution control policy in the USA." in J.S. Shortle and D.G. Abler (eds.), Environmental Policies for Agricultural Pollution Control. Oxfordshire, UK: CAB International.

Ross and Associates Environmental Consulting, Ltd (2000) "Lower boise river effluent trading demonstration project: summary of participant recommendations for a trading framework." Report prepared for the Idaho Division of Environmental Quality. Retrieved from the internet on January 2, 2010 at http://yosemite.epa.gov/r10/oi.nsf/Webpage/Lower+Boise+River+Effluent+Trading +Demonstration+Project/.

Segerson, K. (1988) "Uncertainty and incentives for nonpoint pollution control." Journal of Environmental Economics and Management, 15, 87-98.

Shortle, J.S. and J.W. Dunn (1986) "The relative efficiency of agricultural source water pollution control policies." American Journal of Agricultural Economics, 68(3), 668-677.

Shortle, J.S. and R.D. Horan (2001) "The economics of nonpoint pollution control." Journal of Economic Surveys, 15(3), 255-289.

Shortle, J.S. and R.D. Horan (2005) "When two wrongs make a right: second best point-nonpoint trading ratios." American Journal of Agricultural Economics, 87(2), 340-352.

Tomasi, T., K. Segerson, and J. Braden (1994) "Issues in the design of incentive schemes for nonpoint source pollution control." In C. Dosi and T. Tomasi (eds.), Nonpoint source pollution regulation: issues and analysis. Kluwer Academic Publishers, Dordrecht, Germany.

United States Environmental Protection Agency (EPA) (2005) National water quality report. EPA, Washington, D.C. Retrieved from the internet on June 14, 2006 at http://www.oaspb.epa.gov/waters/national_ rept.control\# IMP_STATE.

United States Environmental Protection Agency (EPA) (2003) "Water quality trading policy: Issuance of final policy." Federal Register, 68(8), 1608-1613. 
United States Environmental Protection Agency (EPA) (2011a) Water: Total maximum daily loads (303d). EPA, Washington, D.C. Retrieved from the internet on October 11, 2011, at http://water.epa.gov/lawsregs/lawsguidance/cwa/tmdl/glossary.cfmt.

United States Environmental Protection Agency (EPA) (2011b) Watershed assessment, tracking, and environmental results. EPA, Washington, D.C. Retrieved from the internet on September 29, 2011, at http://iaspub.epa.gov/waters10/attains nation cy.control.

Varian, H.R. (1992) Microeconomic analysis, Third Edition. W.W. Norton and Company, NY.

Vossler, C.A., G.L. Poe, W.D. Schulze, and K. Segerson (2006) "Communication and incentive mechanisms based on group performance: an experimental study of nonpoint pollution control." Economic Inquiry, 44(4), 599-613.

Wellisch, D. (1994) "Interregional spillovers in the presence of perfect and imperfect household mobility." Journal of Public Economics, 55, 167-184.

Woodward, R.T. and R.A. Kaiser (2002) "Market structures for U.S. water quality trading." Review of Agricultural Economics, 24(2), 366-383. 
Table 1: Parameter Values for Simulations of First- and Second-Best Scenarios.

\begin{tabular}{ccc}
\hline Parameter & Initial Value & Alternative Value \\
$\bar{A}$ & 150 & 150 \\
$\tau_{1}$ & 1 & 0.95 \\
$\tau_{2}$ & 0.95 & 1 \\
$\tau_{P}$ & 1 & 1 \\
$N B_{1}^{A l t}=N B_{2}^{A l t}$ & 100 & 100 \\
$\pi_{1}=\pi_{2}$ & 2 & 2 \\
\hline
\end{tabular}


Table 2: Results for Initial Simulation of First- and Second-Best Scenarios.

\begin{tabular}{lcc}
\hline Variable & First-Best & Second-Best \\
\hline$\beta_{1}$ & & \\
$\beta_{2}$ & 0.49 & 0.49 \\
$\bar{a}_{1}$ & 0.51 & 0.48 \\
$\bar{a}_{2}$ & 36.93 & 27.51 \\
$\bar{a}_{P}$ & 24.42 & 25.69 \\
$a_{1}$ & 89.87 & 98.08 \\
$a_{2}$ & 79.00 & 76.93 \\
$a_{P}$ & 54.26 & 56.92 \\
$\rho$ & 19.46 & 19.01 \\
Profit & 38.91 & 38.01 \\
Profit $_{2}$ & 100 & 416.26 \\
Profit $P$ & 100 & 100 \\
$\mu_{1}$ & $-3,118.20$ & $-3,367.16$ \\
$\mu_{2}$ & 0.35 & 1.06 \\
$\lambda$ & 0.35 & 0.52 \\
Total Cost to Regulator $\left(\left(1-\beta_{1}\right) C_{1}(\cdot)+\left(1-\beta_{2}\right) C_{2}(\cdot)\right)$ & 13.73 & 19.63 \\
& & \\
\end{tabular}


Table 3: Results for Alternative Simulation of First- and Second-Best Scenarios.

\begin{tabular}{lcc}
\hline Variable & First-Best & Second-Best \\
\hline & & \\
$\beta_{1}$ & 0.49 & 0.48 \\
$\beta_{2}$ & 0.51 & 0.52 \\
$\bar{a}_{1}$ & 35.65 & 36.20 \\
$\bar{a}_{2}$ & 26.21 & 25.70 \\
$\bar{a}_{P}$ & 89.93 & 89.91 \\
$a_{1}$ & 76.64 & 77.75 \\
$a_{2}$ & 57.49 & 56.50 \\
$a_{P}$ & 19.70 & 19.64 \\
$\rho$ & 39.40 & 39.27 \\
Profit & 100 & 100 \\
rrofit $_{2}$ & 100 & 100 \\
Profit & $-3,155.10$ & $-3,145.35$ \\
$\mu_{1}$ & 0.39 & 0.41 \\
$\mu_{2}$ & 0.39 & 0.41 \\
$\lambda$ & 15.46 & 16.23 \\
Total Cost to Regulator $\left(\left(1-\beta_{1}\right) C_{1}(\cdot)+\left(1-\beta_{2}\right) C_{2}(\cdot)\right)$ & $2,573.37$ & $2,591.08$ \\
& & \\
\hline
\end{tabular}

